\title{
ON A CERTAIN CLASS OF ISOTHERMIC SURFACES
}

BY

\section{ARCHER E. YOUNG*}

\section{§1. Introduction.}

If a surface, referred to its asymptotic lines, has its linear element in the form

$$
\overline{d s}^{2}=\lambda\left(d u_{1}^{2}+2 \cos \theta d u_{1} d v_{1}+d v_{1}^{2}\right),
$$

it has the characteristic property that its asymptotic lines form a net-work of infinitesimal rhombuses. $\dagger$ Another class of surfaces referred to lines of curvature may have their linear elements put into the form

$$
\overline{d s}^{2}=\lambda\left(d u^{2}+d v^{2}\right)
$$

These surfaces, named isothermic by DARBoux, $\ddagger$ have upon them a net-work of infinitesimal squares formed by the lines of curvature.

These two classes are not exclusive and we have undertaken in this paper to discuss analytically those surfaces which belong to both, with a view of pointing out a general method for their determination and of showing with what comparative ease certain new isothermic surfaces may be obtained.

It appears in $\S 2$ that the lines on the Gauss sphere which are the spherical images of the lines of curvature on the surfaces are isothermal, and hence it may be said that we are seeking those isothermic surfaces which have an isothermal spherical representation of their lines of curvature. It appears at the close of $\S 2$ that the general problem may be reduced to the simultaneous solution of two differential equations, the general solution of one being known, and the other being the GaUss equation for the sphere referred to isothermal lines.

The problem is so general that it is necessary at this point to particularize. We have therefore confined ourselves to a particular solution of the general equations.

The discussion of the particular solution begins in $\S 3$. We give there the first and third differential forms of the resulting surfaces. The surfaces them-

* Presented to the Society (Chicago), March 30, 1907.

$\dagger$ Pseudospherical surfaces are included in this class.

† Théorie der surfaces, vol. 2, p. 246. 
selves are considered in $\S \S 4-7$. In $\S 8$ are considered the other isothermic surfaces which have the same spherical representation of their lines of curvature respectively as those of the four preceding articles. In $\S 9$ it is shown that the general form, under which the linear elements of the surfaces of $\$ \$ 4-7$ come, is characteristic of them.

\section{§2. Analytical discussion of the general problem.}

Suppose $S$ is a surface which has its linear element in the form (I) when referred to asymptotic lines. The equation * of the lines of curvature reduces to

$$
d u_{1}^{2}-d v_{1}^{2}=0 \text {. }
$$

If we take the lines of curvature for lines of reference by writing $d u_{1}+d v_{1}=d u$, $d u_{1}-d v_{1}=d v,(\mathrm{I})$ becomes

$$
\overline{d s^{2}}=\frac{\lambda}{2}\left[(1+\cos \theta) d u^{2}+(1-\cos \theta) d v^{2}\right],
$$

where $\lambda$ and $\theta$ are now functions of the new parameters $u$ and $v$.

The necessary and sufficient condition that the parameter-lines in $(\mathrm{I})^{\prime}$, that is, the lines of curvature, be isothermal, is that $\tan \theta / 2=f(u) / e(v)$.

Now since $\tan \theta / 2=\sqrt{-r_{2} / r_{1}}$, where $r_{1}$ and $r_{2}$ are the principal radii of curvature, we conclude: The necessary and sufficient condition that a surface $S$ whose linear element takes the form $(\mathrm{I})$ when referred to asymptotic lines shall be isothermic is that the ratio of the principal radii of curvature be equal to a function of one of the parameters divided by a function of the other, the lines of reference being lines of curvature on the surface.

The relations existing between the principal radii of curvature and the functions appearing in the first three quadratic forms for a surface show that the surfaces which we seek have as their first and third fundamental forms, when referred to lines of curvature,

$$
\begin{aligned}
& \overline{d s^{2}}=\lambda_{1}\left(\begin{array}{c}
d u^{2} \\
-L_{1}^{\tau^{-}}+ \\
V_{1}
\end{array}\right), \\
& \text { (III) } \overline{d \sigma^{2}}=\lambda\left(\frac{d u^{2}}{U}+\frac{d v^{2}}{V}\right) \text {, }
\end{aligned}
$$

where $U, U_{1}$ and $V, V_{1}$ are functions of $u$ and $v$ respectively.

Our problem then may be reduced to that of finding all surfaces which have (I) and (III) for their first and third forms when referred to lines of curvature. Since, as we have shown, $r_{2} / r_{1}=U_{2} / V_{2}$, where $U_{2}$ and $V_{2}$ are respectively functions of $u$ and $v$, we may write $r_{2}=\mu U_{2}, r_{1}=\mu V_{2}$, and then the CodazzI equations $\dagger$ become

* Branchi, Differentialgeometrie, Lukat's edition, p. 99.

† BranchI, loc. cit., p. 135. 


$$
\begin{aligned}
& U_{2} \frac{\partial \log \mu}{\partial v}=\left(V_{2}-U_{2}\right) \frac{\partial \log \sqrt{\lambda}}{\partial v}, \\
& V_{2} \frac{\partial \log \mu}{\partial u}=\left(U_{2}-V_{2}\right) \frac{\partial \log \sqrt{\lambda}}{\partial u}
\end{aligned}
$$

where $\lambda$ is the function appearing in (III).

If $V_{2}=U_{2}=$ const., the corresponding surfaces are spheres.

If $V_{2}=-U_{2}=$ const., then $r_{1}=-r_{2}$, and the surfaces are minimal. All minimal surfaces are solutions of the problem.*

If $V_{2}$ reduces to a constant, the surfaces are found, by a discussion similar to that of $\S 4$, to be surfaces of revolution. All surfaces of revolution are, as is well known, solutions of the problem.

If we exclude these exceptional cases, the equations (1) may be reduced by a simple change of parameters $\dagger$ to

$$
\begin{aligned}
& u \frac{\partial \log \mu}{\partial v}=(v-u) \frac{\partial \log v^{\bar{\lambda}}}{\partial v}, \\
& v \frac{\partial \log \mu}{\partial u}=(u-v) \frac{\partial \log v \bar{\lambda}}{\partial u},
\end{aligned}
$$

Eliminating $\mu$, we have

$$
\frac{\partial}{\partial u}\left(\frac{v-u}{u} \frac{\partial \log \sqrt{\bar{\lambda}}}{\partial v}\right)=\frac{\partial}{\partial v}\left(\frac{u-v}{v} \frac{\partial \log v^{\prime} \bar{\lambda}}{\partial u}\right) ;
$$

and this, if we replace $u^{2}$ by $1 / u$ and $v^{2}$ by $1 / v$, becomes

$$
(u-v) \frac{\partial^{2} \log v \bar{\lambda}}{\partial u \partial v}-\frac{1}{2} \frac{\partial \log \imath \bar{\lambda}}{\partial u}+\frac{1}{2} \frac{\partial \log v \bar{\lambda}}{\partial v}=0
$$

an equation whose general solution is known. $\ddagger$

The problem then, in its general form, is reduced to the determination of those forms of the general solution of (3)' which will satisfy the Gauss equation for the sphere,

$$
2 U \frac{\partial^{2} \log \sqrt{\lambda}}{\partial u^{2}}+2 V \frac{\partial^{2} \log \sqrt{\lambda}}{\partial v^{2}}+U^{\prime} \frac{\partial \log v \bar{\lambda}}{\partial u}+V^{\prime} \frac{\partial \log v^{\prime} \bar{\lambda}}{\partial v}+2 \lambda=0,
$$

where $U$ and $V$ are arbitrary functions of $u$ and $v$ respectively.

As we can carry the problem in its general form no further, we will take a particular solution of (3), and in treating it, explain the general method to be followed, from this point on, in the determination of these surfaces.

* DARBoux, loc. cit., vol. 1, p. 313.

$\dagger$ In the new parameters $r_{2}=\mu u, r_{1}=\mu v$, and (I) and (III) have the same general form.

$\ddagger$ Darboux, loc. oit., vol. 2, p. 69.

'Truns. Am. Muth. Soc. 6 
§3. A particular solution of the fundamental equations.

Combining the two simple solutions of (3),

we have

$$
\log \sqrt{\lambda}=\log \left(\frac{u+v}{u v}\right)^{l}, \quad \text { and } \quad \log \sqrt{\lambda}=\log \left(\frac{v-u}{u v}\right)^{k},
$$

$$
\log \sqrt{\lambda}=\log \left[\left(\frac{v-u}{u v}\right)^{k}\left(\frac{v+u}{u v}\right)^{\imath}\right] \text {. }
$$

Substituting in (2) and integrating, we find

and hence

$$
\mu=\frac{(v u)^{k+l}}{(v+u)^{2 l}},
$$
(I) $\overline{d s}^{2}=\frac{(v-u)^{2 k}}{(v+u)^{2 l}}\left(\frac{u^{2} d u^{2}}{U}+\frac{v^{2} d v^{2}}{V}\right)$,
(III) $\overline{d \sigma^{2}}=\left(\frac{v-u}{u v}\right)^{2 k}\left(\frac{v+u}{u v}\right)^{2 l}\left(\frac{d u^{2}}{U}+\frac{d v^{2}}{V}\right)$

where the possible values of the constants $k$ and $l$ and the forms of the functions $U$ and $V$ are to be determined by equation (4).

Substituting in (4) the value of $\lambda$ given in (III), we find ${ }^{*}$ as the possible values for $k$ and $l$

$$
\begin{array}{ll}
\left(1^{\circ}\right)\left\{\begin{array}{lll}
l=0 & & k=0 \\
k=\frac{1}{2} & \text { or } & l=\frac{1}{2}
\end{array}\right\}, & \left(2^{\circ}\right)\left\{\begin{array}{lll}
l=0 & & k=0 \\
k=-1 & \text { or } & l=-1
\end{array}\right\}, \\
\left(3^{\circ}\right)\left\{\begin{array}{lll}
l=\frac{1}{2} & & l=-1 \\
k=\frac{1}{2} & \text { or } & k=-1
\end{array}\right\}, & \left(4^{\circ}\right)\left\{\begin{array}{lll}
l=-1 & l=\frac{1}{2} \\
k=\frac{1}{2} & \text { or } & k=-1
\end{array}\right\} .
\end{array}
$$

The corresponding forms for the linear elements on the sphere and surfaces are respectively

$$
\left(1^{\circ}\right)^{\prime}{\overline{d s^{2}}}^{2}=(v-u)\left(\frac{u^{2} d u^{2}}{U}+\frac{v^{2} d v^{2}}{V}\right), \quad \overline{d s^{2}}=\frac{1}{(v+u)}\left(\frac{u^{2} d u^{2}}{U}+\frac{v^{2} d v^{2}}{V}\right) ;
$$$$
\left(2^{\circ}\right) \overline{d \sigma^{2}}=\left(\frac{u v}{v-u}\right)^{2}\left(\frac{d u^{2}}{U}+\frac{d v^{2}}{V}\right), \quad \overline{d \sigma^{2}}=\left(\frac{u v}{v+u}\right)^{2}\left(\frac{d u^{2}}{U}+\frac{d v^{2}}{V}\right) \text {; }
$$$$
\left(2^{\circ}\right)^{\prime} \overline{d s^{2}}=\frac{1}{(v-u)^{2}}\left(\frac{u^{2} d u^{2}}{U}+\frac{v^{2} d v^{2}}{V}\right), \quad \overline{d s}^{2}=(v+u)^{2}\left(\frac{u^{2} d u^{2}}{U}+\frac{v^{2} d v^{2}}{V}\right) ; .
$$

\footnotetext{
* The work is similar to that of $\& 4$.
} 
$\left(3^{\circ}\right)$

$$
\begin{array}{ll}
\overline{d \sigma^{2}}=\left(\frac{v^{2}-u^{2}}{u^{2} v^{2}}\right)\left(\frac{d u^{2}}{U}+\frac{d v^{2}}{V}\right), & \overline{d \sigma^{2}}=\left(\frac{u^{2} v^{2}}{v^{2}-u^{2}}\right)^{2}\left(\begin{array}{c}
d u^{2} \\
U \\
U
\end{array} \frac{d v^{2}}{V}\right) \\
\overline{d s}^{2}=\left(\frac{v-u}{v+u}\right)\left(\frac{u^{2} d u^{2}}{U}+\frac{v^{2} d v^{2}}{V}\right), & \overline{d s^{2}}=\left(\frac{v+u}{v-u}\right)^{2}\left(\frac{u^{2} d u^{2}}{U}+\frac{v^{2} d v^{2}}{V}\right) ;
\end{array}
$$$$
\overline{d \sigma^{2}}=\left(\frac{v-u}{u v}\right)\left(\frac{u v}{v+u}\right)^{2}\left(\frac{d u^{2}}{U^{-}}+\frac{d v^{2}}{V}\right),
$$

$$
\begin{aligned}
& {\overline{d \sigma^{2}}}^{2}\left(\frac{v+u}{u v}\right)\left(\frac{u v}{v-u}\right)^{2}\left(\frac{d u^{2}}{U}+\frac{d v^{2}}{V}\right) ; \\
& \overline{d s}^{2}=(v-u)(v+u)^{2}\left(\frac{u^{2} d u^{2}}{U}+\frac{v^{2} d v^{2}}{V}\right), \\
& \overline{d s}^{2}=\frac{1}{(v+u)(v-u)^{2}}\left(\frac{u^{2} d u^{2}}{U}+\frac{v^{2} d v^{2}}{V}\right),
\end{aligned}
$$

where $U$ and $V$ are written in the place of functions which differ in the several cases but are easily determined.

As is seen by a simple change of parameters, the lines of reference on the sphere are the same for both forms of $\left(1^{\circ}\right)$ and the first of $\left(3^{\circ}\right)$; for both forms of $\left(2^{\circ}\right)$ and the last of $\left(3^{\circ}\right)$; and finally, for both forms of $\left(4^{\circ}\right)$. We have therefore only three distinct isothermal systems on the sphere.

The first expression in $\left(1^{\circ}\right)^{\prime}$ is characteristic of the quadrics, ${ }^{*}$ and the second of their associates, $\dagger$ while the first of $\left(2^{\circ}\right)^{\prime}$ is characteristic of the BonNET surfaces* and the second of their associates. $\dagger$ The surfaces corresponding to the other linear elements are new and require more careful consideration.

\section{§4. Surfaces corresponding to $\left(4^{\circ}\right)^{\prime}$.}

Replacing $U$ and $V$ by the values found we have

$$
\begin{aligned}
& \overline{d s}^{2}=(v-u)(v+u)^{2}\left(\frac{d u^{2}}{\alpha_{0} u^{2}-u+\alpha_{2}}-\frac{d v^{2}}{\alpha_{0} v^{2}-v+\alpha_{2}}\right), \\
& \overline{d s}_{1}^{2}=\frac{1}{(v-u)(v+u)^{2}}\left(\frac{d u^{2}}{\alpha_{0} u^{2}-u+\alpha_{2}}-\frac{d v^{2}}{\alpha_{0} v^{2}-v+\alpha_{2}}\right) .
\end{aligned}
$$

In order to determine whether these forms are characteristic of the corresponding surfaces, we shall consider the form

$$
\overline{d s}^{2}=(v-u)(v+u)^{2}\left(\frac{d u^{2}}{U}+\frac{d v^{2}}{V}\right),
$$

* Bonnet, Journal de L'Ecole Polytechnique, vol. 42 (1867), pp. 121-151.

$\dagger$ A. E. Young, On certain isothermic surfaces, Transactions of the American Mathematical Society, vol. 8 (1907), p. 419. 
and determine for what values of the functions $U$ and $V$, this expression may be the linear element of a surface referred to its lines of curvature. Writing the GaUss and CodazzI equations in the form*

$$
\frac{\partial M}{\partial v}-\frac{\partial N}{\partial u}=P Q, \quad \frac{\partial P}{\partial v}-M Q=0, \quad \frac{\partial Q}{\partial u}-N P=0,
$$

we derive * from them the following

$$
\frac{\partial\left(P^{2}+M^{2}\right)}{\partial v}=2 M \frac{\partial N}{\partial u}, \quad \frac{\partial\left(\cdot Q^{2}+N^{2}\right)}{\partial u}=2 N-\frac{\partial M}{\partial v} .
$$

Applying these to the above linear element, since

$$
M=-\sqrt{\frac{V}{U}}\left(\frac{3 v-u}{2\left(v^{2}-u^{2}\right)}\right), \quad N=\sqrt{\frac{U}{V}}\left(\frac{v-3 u}{2\left(v^{2}-u^{2}\right)}\right),
$$

we have on integrating (6),

$$
\begin{aligned}
& P^{2}=\frac{1}{4 U}\left[U^{\prime} \frac{(3 v-5 u)}{v^{2}-u^{2}}-\frac{(3 v-u)^{2}(U+V)}{\left(v^{2}-u^{2}\right)^{2}}+U_{1}\right], \\
& Q^{2}=\frac{-1}{4 V}\left[V^{\prime} \frac{(3 u-5 v)}{\left(v^{2}-u^{2}\right)^{2}}+\frac{(3 u-v)^{2}(U+V)}{\left(v^{2}-u^{2}\right)^{2}}+V_{1}\right],
\end{aligned}
$$

where $U_{1}$ and $V_{1}$ are new functions introduced by the integration. Squaring both sides of the first equation in (5), substituting the values found for $P^{2}, Q^{2}$, $M$ and $N$, and removing the common factor $(u+v)^{2}$, we have

$$
\begin{aligned}
3\left(9 v^{2}\right. & \left.-14 u v+9 u^{2}\right)(U+V)^{2}+\left[3(v-u)(v-3 u)(u-3 v)\left(U^{\prime}-V^{\prime}\right)\right. \\
& \left.-(v-u)^{2}\left\{(3 v-u)^{2} V_{1}-(v-3 u)^{2} U_{1}\right\}\right](U+V)+(v-u)^{2}\left[U^{\prime}(v-3 u)\right. \\
& \left.+V^{\prime}(3 v-u)\right]^{2}+(v-u)^{2}\left[(3 v-5 u) U^{\prime}\right. \\
& \left.+\left(v^{2}-u^{2}\right) U_{1}\right]\left[(3 u-5 v) V^{\prime}+V_{1}\left(v^{2}-u^{2}\right)\right]=0 .
\end{aligned}
$$

Replacing $u$ and $v$ by $\alpha$, where $\alpha$ is supposed to be some value for which all the functions are analytic, $\nmid$ we have from (7)

$$
U(\alpha)+V(\alpha)=0 \text {. }
$$

Writing $U(\alpha)=-V(\alpha)=f(\alpha)$, replacing $u$ and $v$ in (7) by $\alpha+h$ and $\alpha-h$ respectively, and expanding the functions in powers of $h$, we have

* Bonnet, loc. cit., p. 133.

† The question as to whether this assumption limits our results we shall not attempt to settle in this paper. 


$$
\begin{aligned}
& 48 h^{2}\left(\alpha^{2}+8 h^{2}\right)\left(f^{\prime}+\frac{f^{\prime \prime \prime} h^{2}}{\underline{3}}+\frac{f^{\mathrm{v}} h^{4}}{\underline{5}}+\cdots\right)^{2}+96 h^{2}\left(4 h^{2}-\alpha^{2}\right) \\
& \quad \times\left(f^{\prime}+\frac{f^{\prime \prime \prime} h^{2}}{\underline{L}}+\cdots\right)\left(f^{\prime}+\frac{f^{\prime \prime \prime} h^{2}}{\underline{3}}+\cdots\right)-32 h^{3}\left[(\alpha-2 h)^{2}\right. \\
& \left.\quad \times\left(V_{1}-V_{1}^{\prime} h+\cdots\right)-(\alpha+2 h)^{2}\left(U_{1}+U_{1}^{\prime} h+\cdots\right)\right]\left(f^{\prime}+\frac{f^{\prime \prime \prime} h^{2}}{\lfloor 3}+\cdots\right) \\
& \quad+64 h^{2}\left[\alpha\left(f^{\prime}+\frac{f^{\prime \prime \prime} h^{2}}{2}+\cdots\right)+2 h^{2}\left(f^{\prime \prime}+\frac{f^{\mathrm{vv}} h^{2}}{\lfloor 3}+\cdots\right)\right]^{2} \\
& \quad+16 h^{2}\left[(\alpha+4 h)\left(f^{\prime}+f^{\prime \prime} h+\cdots\right)+2 \alpha h\left(U_{1}+V_{1}^{\prime} h+\cdots\right)\right] \\
& \times\left[(\alpha-4 h)\left(-f^{\prime}+f^{\prime \prime} h-\frac{f^{\prime \prime \prime} h^{2}}{2}+\cdots\right)+2 \alpha h\left(V_{1}-V_{1}^{\prime} h+\cdots\right)\right]=0 .
\end{aligned}
$$

The coefficients of all powers of $h$ up to the third inclusive vanish, and the coefficient of $h^{4}$ arranged in factors and equated to zero gives

$$
\left(\alpha f^{\prime \prime}+8 f^{\prime}+2 \alpha U_{1}\right)\left(\alpha f^{\prime \prime}+8 f^{\prime}+2 \alpha V_{1}\right)=0 \text {. }
$$

Making use of the equations obtained by equating the coefficients of $h^{5}$ and $h^{6}$ to zero, we show without difficulty that the most general forms for $U$ and $V$ are

$$
\begin{aligned}
& U=\alpha_{0} u^{3}+\alpha_{1} u^{2}+a_{2} u+\alpha_{3}, \\
& V=-\alpha_{0} v^{3}-\alpha_{1} v^{2}-\alpha_{2} v-\alpha_{3} .
\end{aligned}
$$

These values satisfy (7) only when $\alpha_{0}$ vanishes, and hence the forms $\left(4^{\circ}\right)^{\prime}$ given at the beginning of this article are characteristic of the surfaces found.

Their cartesian coördinates are obtained in the next article.

\section{§ 5. Surfaces corresponding to $\left(4^{\circ}\right)^{\prime}$.}

If $\alpha_{0} \neq 0$,* replacing $u$ and $v$ by $1 / u, 1 / v$ respectively, we may write the first expressions in $\left(4^{\circ}\right)$ and $\left(4^{\circ}\right)^{\prime}$ in the forms

$$
\overline{d \sigma^{2}}=\left(\frac{u-v}{(u+v)^{2}}\right)\left(\frac{d u^{2}}{a^{2}-\left(\frac{2 u+1}{2}\right)^{2}}-\frac{d v^{2}}{a^{2}-\left(\frac{2 v+1}{2}\right)^{2}}\right)
$$

$$
{\overline{d s^{2}}}^{2}=\frac{(u-v)(u+v)^{2}}{u^{3} v^{3}}\left(\frac{d u^{2}}{u^{2}\left[a^{2}-\left(\frac{2 u+1}{2}\right)^{2}\right]}-\frac{d v^{2}}{v^{2}\left[a^{2}-\left(\frac{2 v+1}{2}\right)^{2}\right]}\right)
$$

* Corresponding to $a_{0}=0$, there exists another surface and its associate. These we have lound but do not consider here. 
If $X, Y, Z$ are the direction cosines* of the normal to the surface, then

$$
\begin{aligned}
& X=-\frac{\sqrt{2 a\left[1-\left(\frac{2 u+1}{2 a}\right)\right]\left[1-\left(\frac{2 v+1}{2 a}\right)\right]}}{(u+v)}, \quad Z=\frac{u+v+2}{(u+v)} \\
& Y=i \frac{\sqrt{2 a\left[1+\left(\frac{2 u+1}{2 a}\right)\right]\left[1+\left(\frac{2 v+1}{2 a}\right)\right]}}{(u+v)},
\end{aligned}
$$

The values of the principal radii of curvature in these parameters are

$$
r_{1}=\frac{(v+u)^{2}}{v(v u)^{\frac{3}{2}}}, \quad r_{2}=\frac{(v+u)^{2}}{u(v u)^{\frac{3}{2}}}
$$

Substituting in the formulas

$$
\frac{\partial x}{\partial u}=r_{2} \frac{\partial X}{\partial u}, \quad \frac{\partial x}{\partial v}=r_{1} \frac{\partial X}{\partial v}
$$

etc., and integrating we have for the cartesian coördinates of the surfaces corresponding to (I)

$$
\begin{aligned}
& x=\frac{\sqrt{\frac{a}{2} \sqrt{\left(a-\frac{(2 u+1)}{2}\right)\left(a-\frac{(2 v+1)}{2}\right)}}[(u+v)(1-2 a)}{3\left(\frac{2 v+1}{2}\right)^{2}(u v)^{\frac{3}{2}}}\left[-4 u v-(2 a-1)^{2}\right], \\
& \left.y=i \frac{\sqrt{\frac{a}{2} \sqrt{\left(a+\frac{(2 u+1)}{2}\right)\left(a+\frac{(2 v+1)}{2}\right)}}[-(u+v)(2 a+1)}{3\left(\frac{2 a+1}{2}\right)^{2}(u v)^{\frac{3}{2}}}+4 u v+(2 a+1)^{2}\right], \\
& z=\frac{4 a}{3(u v)^{\frac{3}{2}}} .
\end{aligned}
$$

The associates $\dagger$ of these surfaces, corresponding to the linear element

$$
\overline{d s}^{2}=\frac{u^{3} v^{3}}{(u-v)(u+v)^{2}}\left(\frac{d u^{2}}{u^{2}\left[a^{2}-\left(\frac{2 u+1}{2}\right)^{2}\right]}-\frac{d v^{2}}{v^{2}\left[a^{2}-\left(\frac{2 v+1}{2}\right)^{2}\right]}\right),
$$

have the same direction cosines as the former and hence their cartesian coördinates could be easily obtained. The surfaces (10) have positive total curvature and their associates negative.

* Considered as coördinates of a point on the Gauss sphere, these may be obtained by the proper change of parameter-lines from their values when the sphere is referred to asymptotic lines.

† Dakboux, loc. cit., p. 243. 
The equations (9) show that the lines on the sphere which are the images of the lines of curvature on the surfaces are two systems of spherical ellipses which, however, are not confocal as in the case of the quadrics.

\section{§6. An infinity of surfaces having at least one set of the lines of curvature plane.}

The functions $U$ and $V$ which appear in the second expression of $\left(3^{\circ}\right)^{\prime}$, as we determined them in the work above took simple forms, but the same expression with a more general value for one or the other of these functions represents other isothermic surfaces (upon which, however, the asymptotic lines do not form a net-work of rhombuses). We will show this as follows:

Writing the second expression of $\left(3^{\circ}\right)^{\prime}$ in the form

$$
\overline{d s}^{2}=\left(\frac{\sqrt{v}+\sqrt{u}}{\sqrt{v}-v \bar{u}}\right)\left(\frac{d u^{2}}{4 u U}+\frac{d v^{2}}{4 v V}\right),
$$

we find that the functions $M$ and $N$ formed for this are the same respectively as those formed for

$$
\overline{d s}^{2}=\frac{1}{(v-u)^{2}}\left(\frac{d u^{2}}{U}+\frac{d v^{2}}{V}\right)
$$

and hence from (5) it appears that $P, Q, U$ and $V$ will have the same values respectively in the two cases.

We know therefore that the most general form for the first expression above is

$$
\overline{d s}^{2}=\left(\frac{\sqrt{u}+\sqrt{v}}{\sqrt{u}-\sqrt{\bar{v}}}\right)\left(\frac{d u^{2}}{4 u\left(\alpha u^{2}+\alpha^{\prime} u+\alpha^{\prime \prime}\right)}+\frac{d v^{2}}{4 v V}\right),
$$

where $V$ is an arbitrary function of $v$.

The cartesian coördinates * for these surfaces are, if $\alpha=-1$ and $\alpha^{\prime \prime}=0$,

$$
\begin{aligned}
& x=\frac{2 \sqrt{v} \sqrt{\alpha^{\prime}-u}}{\alpha^{\prime}(\sqrt{v}-\sqrt{u})}+\int \frac{d u}{2 u \sqrt{\alpha^{\prime}-u}}, \\
& y=-\frac{\sqrt{v-\alpha^{\prime}}}{\alpha^{\prime}}\left(\frac{\sqrt{v}+\sqrt{u}}{\sqrt{v}-\sqrt{u}}\right) \cos V_{3}+\int \frac{\sqrt{v-\alpha^{\prime}}}{2 \alpha^{\prime} v} \cos V_{3} d v, \\
& z=-\frac{\sqrt{v-\alpha^{\prime}}}{\alpha^{\prime}}\left(\frac{\sqrt{v}+\sqrt{u}}{\sqrt{v}-\sqrt{u}}\right) \sin V_{3}+\int \frac{\sqrt{v-\alpha^{\prime}}}{2 \alpha^{\prime} v} \sin V_{3} d v,
\end{aligned}
$$

where

$$
V_{3}=-\int \frac{\alpha_{1} \sqrt{v^{2}-\alpha^{\prime} v-V} d v}{2\left(v^{2}-\alpha^{\prime} v\right) v \bar{V}^{-}}
$$

* We obtain these in the nsual way since we know $X, Y, Z$, which are the same for surfaces corresponding to either linear element. 
The lines $v=$ const. on these surfaces lie in planes which envelope a cylindrical surface whose form is perfectly arbitrary depending upon the function $V$.

If we confine ourselves to real values of $u$ and $v$ and of the constants appearing in (11), the corresponding surfaces will have positive total curvature, the equations of their associates (which have negative total curvature) are obtained by replacing $\sqrt{u}$ by $-v \bar{u}$. Hence, in this case, we may say that the surfaces and their associates are defined by the same system of equations. *

If the linear element above reduces to

where

$$
\overline{d s}^{2}=\left(\frac{\sqrt{u}+\sqrt{v}}{\sqrt{u}-\sqrt{\bar{v}}}\right)^{2}\left(\frac{d u^{2}}{4 u\left(\alpha u^{2}+\alpha^{\prime} u+\alpha^{\prime \prime}\right)}+\frac{d v^{2}}{4 v\left(\beta v^{2}+\beta^{\prime} v+\beta^{\prime \prime}\right)}\right)
$$

$$
4(\alpha+\beta)\left(\alpha^{\prime \prime}+\beta^{\prime \prime}\right)-\left(\alpha^{\prime}+\beta^{\prime}\right)^{2}=0,
$$

both sets of the lines of curvature will be plane curves, having as their images on the Gauss sphere the same lines as have the cyclides of DupIN and their associates.

§ 7. Surfaces having the same spherical representation of their lines of curvature as the quadrics.

If we substitute the values found for $U$ and $V$, the first expression in $\left(3^{\circ}\right)^{\prime}$ becomes

(I) $\overline{d s}^{2}=\left(\frac{v-u}{v+u}\right)\left(\frac{u^{2} d u^{2}}{\alpha_{0} u^{6}+\alpha_{1} u^{4}+\alpha_{2} u^{2}+\alpha_{3}}+\frac{v^{2} d v^{2}}{\alpha_{0} v^{6}+\alpha_{1} v^{4}+\alpha_{2} v^{2}+\alpha_{3}}\right)$.

This form $\dagger$ is characteristic of these surfaces, we know, since all surfaces which have their linear elements in this general form have the same spherical representation of their lines of curvature as the quadrics. $\neq$

The cartesian coördinates for the surfaces corresponding to $(\mathrm{I})$ are

$$
\begin{aligned}
& x=\frac{1}{\sqrt{(a-b)(a-c)}} \log \left[\frac{(u+v)\left(\sqrt{a} u+\sqrt{a u^{2}-1}\right)\left(\sqrt{a} v+\sqrt{a v^{2}-1}\right)}{\left[\sqrt{a}(u+v)+\sqrt{a u^{2}-1}+\sqrt{a v^{2}-1}\right]^{2}}\right], \\
& \text { (12) } y=\frac{1}{\sqrt{(\overline{b-a})(\bar{b}-c)}} \log \left[\frac{(u+v)\left(\sqrt{b} u+\sqrt{\left.b u^{2}-1\right)}\left(\sqrt{b} v+\sqrt{\left.b v^{2}-1\right)}\right.\right.}{\left[\sqrt{b}(u+v)+\sqrt{b u^{2}-1}+\sqrt{\left.b v^{2}-1\right]^{2}}\right.}\right] \text {, } \\
& z=\frac{1}{\sqrt{\prime}(c-a)(c-b)} \log \left[\frac{(u+v)\left(\sqrt{c} u+\sqrt{c u^{2}-1}\right)\left(\sqrt{c} v+\sqrt{c v^{2}}-1\right)}{\left[\sqrt{c(u+v)+\sqrt{c u^{2}-1}+\sqrt{\left.c v^{2}-1\right]^{2}}}\right]},\right.
\end{aligned}
$$

* These surfaces are included in a general solution by Darboux, loc. cit., vol. 4, p. 217. $\dagger$ The most general form is really

$$
\overline{d s^{2}}=\left(\frac{v-u}{v+u}\right)\left(\frac{\left(c_{0} u^{2}+c_{1}\right) d u^{2}}{\alpha_{0} u^{6}+\alpha_{1} u^{4}+\alpha_{2} u^{2}+\alpha_{3}}+\frac{\left(c_{0} v^{2}+c_{1}\right) d v^{2}}{\alpha_{0} \eta^{6}+\alpha_{1} v^{4}+\alpha_{2} v^{2}+\alpha_{3}}\right),
$$

where the coustant $c_{1}$ can be taken equal to zero without affecting essentially the form of the surfaces.

$\ddagger$ That is, spherical confocal conics. 
where in (I) $\alpha_{3}=-1$, and $a, b, c$ are the roots in $u^{2}$ of

$$
-u^{6}+\alpha_{2} u^{4}+\alpha_{1} u^{2}+\alpha_{0}=0 \text {. }
$$

These surfaces do not have plane lines of curvature of course. If $u$ and $v$ have like signs, ${ }^{*}$ the surfaces defined by (12) have positive total curvature; otherwise their curvature is negative. One set may be considered the associates of the other. In each set are three surfaces, one from each set corresponding to each of the central quadrics. To each of the non-central quadrics (corresponding to the case where one of the constants $a, b, c$ vanishes) correspond pairs of surfaces whose equations are easily obtained.

In this and the two preceding articles we have determined three new classes of isothermic surfaces and found the cartesian coördinates for the same. We may apply the principle $f$ of inversion to them and thus obtain from each class an entirely new set of isothermic surfaces. If we then apply to the surfaces last found the Bour-Darboux $\ddagger$ theorem, we have again three sets of isothermic surfaces which are distinct from any of the preceding set.

Repeating the processes in the order named we are led $\S$ to a whole system of isothermic surfaces from each of the sets already found. The cartesian coördinates of all of these surfaces are obtained by quadrature from those given in the preceding articles.

\$8. Other isothermic surfaces having the same spherical representation of their lines of curvature as those discussed in the preceding articles.

Case $1^{\circ}$. All surfaces having the same spherical representation of their lines of curvature as those corresponding to

$$
\overline{d s}^{2}=(v-u)(v+u)^{2}\left(\frac{d u^{2}}{\alpha_{0} u^{2}-u+\alpha_{2}}-\frac{d v^{2}}{\alpha_{0} v^{2}-v+\alpha_{2}}\right)
$$

will have for their first form

$$
\overline{d s}^{2}=\left(\frac{E_{1} d u^{2}}{\alpha_{0} u^{2}-u+\alpha_{2}}-\frac{G_{1} d v^{2}}{\alpha_{10} v^{2}-v+\alpha_{2}}\right),
$$

where $E_{1}$ and $G_{1}$ are such functions that the quantities $M$ and $N$ formed $\|$ for this expression are the same as those formed for (I). If the surfaces corresponding to $(\mathrm{I})^{\prime}$ are to be isothermic, then must

$$
E_{1}=\frac{\lambda}{U_{1}}, \quad G_{1}=\frac{\lambda}{V_{1}},
$$

where $\lambda$ is a function of $u$ and $v$.

\footnotetext{
* For $r_{2} / r_{1}=u / v$.

† Darboux, loo. cit., vol. 2, p. 243.

$\ddagger$ See preceding reference.

§ DARBOUX, loc. cit., vol. 2, p. 247.

|l See equations (5) and (6), $\$ 4$.
} 
Making these substitutions in $(\mathrm{I})^{\prime}$ and expressing the condition that $M$ and $N$ are the same respectively for (I) and (I)', we have

$$
\frac{\partial \log v \bar{\lambda}}{\partial v}=\sqrt{\frac{U_{1}}{V_{1}}}\left(\frac{3 v-u}{\left.2 i v^{2}-u^{2}\right)}\right), \quad \frac{\partial \log V \bar{\lambda}}{\partial u}=\sqrt{\frac{V_{1}}{U_{1}}}\left(\frac{v-3 u}{2\left(v^{2}-u^{2}\right)}\right)
$$

and hence

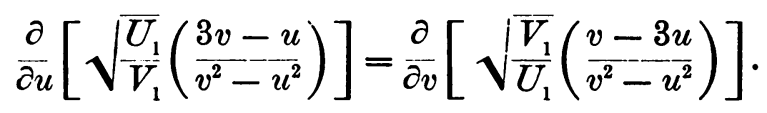

The only values of $U_{1}$ and $V_{1}$ which satisfy this last equation are

$$
U_{1}=c_{0} u^{2}+c_{1}, \quad V_{1}=c_{0} v^{2}+c_{1} .
$$

Substituting these values in (13) and integrating, we obtain for $\lambda$ the expression

$$
\lambda \doteq \frac{\left(u \sqrt{c_{0} v^{2}+c_{1}}+v \sqrt{c_{0} u^{2}+c_{1}}\right)^{2}\left[\left(\sqrt{c_{1} u+}+u \sqrt{c_{0} v^{2}+c_{1}}\right)^{2}-\left(\sqrt{c_{1}} v+v \sqrt{c_{0} u^{2}+c_{1}}\right)^{2}\right]^{3}}{\left(u^{2}-v^{2}\right)\left[( \sqrt { c _ { 1 } } + \sqrt { c _ { 0 } v ^ { 2 } + c _ { 1 } } ) ^ { 2 } \left(\sqrt{\left.\left.c_{1}+\sqrt{c_{0} u^{2}+c_{1}}\right)^{2}-c_{0}^{2} u^{2} v^{2}\right]^{3}}\right.\right.},
$$

and for the linear element

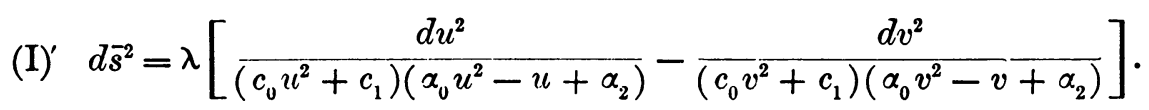

The linear element of the associate of such a surface is obtained by inverting the first factor on the right of $(I)^{\prime}$.

If $c_{0}=0(\mathrm{I})^{\prime}$ reduces to $(\mathrm{I})$, and if $c_{1}=0$ the above expression becomes indeterminate. Going back to equations (13) we find in this case a form which reduces to $(\mathrm{I})$ if the parameters are replaced by their reciprocals. Hence only the general case leads to new surfaces.

The spherical images of the lines of curvature of these new surfaces is the same as that of the others, that is, they are spherical ellipses. Hence the cartesian coördinates of the surfaces are easily obtained by quadratures. The surfaces corresponding to the general form of $(\mathrm{I})^{\prime}$ have positive and their associates negative total curvature.

Case $2^{\circ}$. Following the same method, we find as the most general first fundamental form for isothermic surfaces having the same spherical representation of their lines of curvature as the surfaces of $\S 6$ :

(I) $d s^{2}=\lambda\left(\frac{d u^{2}}{4 u\left(c_{0} u^{2}+c_{1} u+c_{2}\right)\left(a_{0} u^{2}+a_{1} u+a_{2}\right)}+\frac{d v^{2}}{4 v\left(c_{0} v^{2}+c_{1} v+c_{2}\right) V}\right)$, where

$$
\lambda=\frac{(v-u)^{2}\left(c_{0} v+{ }_{2}^{c_{1}}+\sqrt{c_{0}} v^{\prime} \overline{c_{0} v^{2}+c_{1} v+c_{2}}\right)^{2}\left(c_{0} u+\frac{c_{1}}{2}+\sqrt{c_{0}} \sqrt{c_{0} u^{2}+c_{1} u+c_{2}}\right)^{2}}{\left(\sqrt{c_{0}}(v-u)+\sqrt{c_{0} v^{2}+c_{1} v+c_{2}}-\sqrt{c_{0} u^{2}+c_{1} u+c_{2}}\right)^{4}},
$$

or else has the reciprocal value. We have found then, the linear elements for 
the most general class of isothermic surfaces which have same spherical representation of their lines of curvature as the Bonnet surfaces* and their associates. One set of their lines of curvature is always plane, and when the function $V$ has its particular form, both sets are plane. Their cartesian coördinates are obtained by quadrature. The isothermic surfaces first obtained from them by inversion will have one (or both) sets of their lines of curvature spherical. $\dagger$

Case $3^{\circ}$. The most general form for the linear element of isothermic surfaces having the same spherical representation of their lines of curvature as the quadrics, that is, spherical confocal conics, is

(I) $d s^{2}=\lambda\left[\frac{d u^{2}}{\left(c_{0} u^{2}+c_{1} u+c_{2}\right)\left(\alpha_{0} u^{3}+\alpha_{1} u^{2}+\alpha_{2} u+\alpha_{3}\right)}\right.$

where

$$
\left.+\frac{d v^{2}}{\left(c_{0} v^{2}+c_{1} v+c_{2}\right)\left(\alpha_{0} v^{3}+\alpha_{1} v^{2}+\alpha_{2} v+\alpha_{3}\right)}\right],
$$

$\lambda=\frac{\left[\sqrt{c_{0}}(v-u)+\sqrt{c_{0} v^{2}+c_{1} v+c_{2}}-\sqrt{c_{0} u^{2}+c_{1} u+c_{2}}\right]^{2}}{(v-u)\left(2 c_{0} u+c_{1}+2 \sqrt{c_{0}} \sqrt{\left.c_{0} u^{2}+c_{1} u+c_{2}\right)}\left(2 c_{0} v+c_{1}+2 \sqrt{c_{0}} \sqrt{\left.c_{0} v^{2}+c_{1} v+c_{2}\right)}\right.\right.}$,

or else has the reciprocal value. The following cases, which apply as well to the associates of $(I)$, may be noted :

$$
\begin{aligned}
& c_{0} \neq 0, c_{1} \neq 0, c_{2} \neq 0, \\
& c_{0} \neq 0, c_{1}=0, c_{2} \neq 0,
\end{aligned}
$$

$$
c_{0} \neq 0, c_{1}=0, c_{2}=0,
$$

$$
c_{0}=0, c_{1} \neq 0, c_{2}=0 \text { or } \neq 0,
$$

$$
c_{0}=0, c_{1}=0, c_{2}=0 \text {. }
$$

$\left(1^{\circ}\right)$ is the general case.

$\left(2^{\circ}\right)$ gives the quadrics and their associates.

$\left(3^{\circ}\right)$ gives a class not discussed.

$\left(4^{\circ}\right)$ gives the surface of $\S 7$.

$\left(5^{\circ}\right)$ gives the sphere and the associate minimal surface.

§ 9. Discussion of the general form of the linear element which includes all the forms of $\S 3$ as particular cases.

In $\S 2$ we found for what values of the constants and for what forms of the functions the expression

* The BonNet surfaces are the inverses of surfaces of revolution, cones, and cylinders.

† We have shown in a paper soon to be published that all isothermic surfaces having one set of their lines of curvature plane must have the same spherical representation of their lines of curvature as the Bonnet Surfaces and their associates. The surfaces of Case $2^{\circ}$ are therefore identical with this general class, and are the surfaces discussed by DARBoux in his Theorie des Surfaces, Vol. IV., $\mathrm{Ch} . \mathrm{X}$. Treated from this standpoint, the determination of these surfaces is an elementary problem, the solution of which makes unnecessary most of the work of the chapter referred to above. 


$$
\overline{d s}^{2}=\left(\frac{v-u}{u v}\right)^{k}\left(\frac{v+u}{u v}\right)^{2}\left(\frac{d u^{2}}{U}+\frac{d v^{2}}{V}\right)
$$

could be the linear element of the sphere. The linear elements of the surfaces which we then found directly came under the one general form

$$
\overline{d s}^{2}=\frac{(v-u)^{2 k}}{(v+u)^{2 l}}\left(\frac{d u^{2}}{U}+\frac{d v^{2}}{V}\right) .
$$

Before leaving the subject we inquire if the form (I) is characteristic of the surfaces already discussed, or whether there are other surfaces whose linear elements come under the same form when referred to lines of curvature.

We find that for (I)

$$
M=-\sqrt{\frac{V}{U}}\left(\frac{k}{v-u}-\frac{l}{v+u}\right), \quad N=-\sqrt{\bar{V}} \frac{k}{V}\left(\frac{k}{v-u}+\frac{l}{v+u}\right) .
$$

Substituting these values in equations $(6)$ of $\S 4$ and integrating, we find

$$
\begin{aligned}
& P^{2}=-\frac{1}{U}\left[\left(\frac{k}{v-u}-\frac{l}{v+u}\right)^{2}(U+V)+\left(\frac{k^{2}}{v-u}-\frac{l^{2}}{v+u}\right) U^{\prime}+U_{1}\right], \\
& Q^{2}=-\frac{1}{V}\left[\left(\frac{k}{v-u}+\frac{l}{v+u}\right)^{2}(U+V)-\left(\frac{k^{2}}{v-u}+\frac{l^{2}}{v+u}\right) V^{\prime}+V_{1}\right] .
\end{aligned}
$$

The GAUSs equation in (5) with these values substituted gives, upon squaring both sides,

$$
\begin{aligned}
(U+ & V)^{2}\left[\left(\frac{k}{(v-u)^{2}}-\frac{l}{(v+u)^{2}}\right)^{2}-\left(\frac{k^{2}}{(v-u)^{2}}-\frac{l^{2}}{(v+u)^{2}}\right)^{2}\right] \\
& +(U+V)\left[U ^ { \prime } \left\{\left(\frac{k}{v-u}+\frac{l}{v+u}\right) \cdot\left(\frac{k}{(v-u)^{2}}-\frac{l}{(v+u)^{2}}\right)\right.\right. \\
& \left.-\left(\frac{k}{(v-u)}+\frac{l}{v+u}\right)^{2}\left(\frac{k^{2}}{v-u}-\frac{l^{2}}{v+u}\right)\right\}+V^{\prime}\left\{\left(\frac{k}{v-u}-\frac{l}{v+u}\right)\right. \\
& \left.+\left(\frac{l}{(v+u)^{2}}-\frac{k}{(v-u)^{2}}\right)+\left(\frac{k}{v-u}-\frac{l}{v+u}\right)^{2}\left(\frac{k^{2}}{v-u}+\frac{l^{2}}{v+u}\right)\right\} \\
& \left.-U_{1}\left(\frac{k}{v-u}+\frac{l}{v+u}\right)^{2}-V_{1}\left(\frac{k}{v-u}-\frac{l}{v+u}\right)^{2}\right] \\
& +\frac{V^{\prime 2}}{4}\left(\frac{k}{v-u}-\frac{l}{v+u}\right)^{2}+\frac{U^{\prime 2}}{4}\left(\frac{k}{v-u}+\frac{l}{v+u}\right)^{2} \\
& +U^{\prime} V^{\prime}\left[\frac{k^{2}\left(2 k^{2}-1\right)}{2(v-u)^{2}}-\frac{l^{2}\left(2 l^{2}-1\right)}{2(v+u)^{2}}\right] \\
& +\left(U_{1} V^{\prime}-V_{1} U^{\prime}\right)\left(\frac{k^{2}}{v-u}+\frac{l^{2}}{v+u}\right)-U_{1} V_{1}=0 .
\end{aligned}
$$


The coefficient of $(U+V)^{2}$ in this equation will vanish under the following conditions :

$$
\begin{array}{ll}
1^{\circ} . k=0, l= \pm 1, & 2^{\circ} \cdot l=0, k= \pm 1, \\
3^{\circ} . l=k=1, & 4^{\circ} . l=k=-1 .
\end{array}
$$

The coefficient of $(U+V)^{2}$, after clearing of fractions, will be divisible by $(u+v)^{2}$, but not by $(u-v)^{2}$, when

$$
5^{\circ} . l=0 \text { or } \pm 1, k \neq 0 \text { or } \pm 1 \text {. }
$$

Finally, this coefficient will be divisible by $(u-v)^{2}$, but not by $(u+v)^{2}$, when

$$
6^{\circ} . k=0 \text { or } \pm 1, l \neq 0 \text { or } \pm 1 \text {. }
$$

If no one of the six foregoing conditions is fulfilled, replacing $u$ and $v$ by $\alpha$, where $\alpha$ is a value for which all the functions are analytic, we have, from (14), $U(\alpha)+V(\alpha)=0$, and replacing $u$ by $\alpha$ and $v$ by $-\alpha$, and vice versa,

Hence

$$
U(\alpha)+V(-\alpha)=0, \quad U(-\alpha)+V(\alpha)=0 .
$$

$$
U(\alpha)=U(-\alpha)=-V(\alpha)=-V(-\alpha)
$$

or the functions $U$ and $V$ are even functions of $u$ and $v$ respectively. Their odd derivatives must therefore merely change signs with the variables.

If we assume that $U_{1}$ and $V_{1}$ are also even functions, we may divide (14) into two parts, one being composed of terms which merely change their signs, the other of terms which are unaltered when the sign of either variable is changed.

The former of these two parts equated to zero gives

$$
\begin{aligned}
& (U+V)^{2}\left[\frac{\left[k^{2}\left(1-k^{2}\right)-l^{2}\left(1-l^{2}\right)\right] 4 u v\left(u^{2}+v^{2}\right)}{\left(v^{2}-u^{2}\right)^{3}}\right] \\
& +(U+V)\left[\begin{array}{r}
\left\{k^{2}\left(1-k^{2}\right)-l^{2}\left(1-l^{2}\right)\right\}\left\{v U^{\prime}\left(v^{2}+3 u^{2}\right)-u V^{\prime}\left(3 v^{2}+u^{2}\right)\right\} \\
+2 k l\left(l^{2}-k^{2}\right)\left(v^{2}-u^{2}\right)\left(v U^{\prime}+u V^{\prime}\right) \\
\left(v^{2}-u^{2}\right)^{2}
\end{array}\right. \\
& \left.+\frac{2\left(l^{2}-k^{2}\right) u v\left(U_{1}+V_{1}\right)}{\left(v^{2}-u^{2}\right)}\right]+\frac{\left(v^{2}+u^{2}\right) U^{\prime} V^{\prime}\left\{k^{2}\left(2 k^{2}-1\right)-l^{2}\left(2 l^{2}-1\right)\right\}}{\left(v^{2}-u^{2}\right)} \\
& +\frac{\left(k^{2}-l^{2}\right) u v\left(U^{\prime 2}+V^{\prime 2}\right)}{2}+\left(k^{2}-l^{2}\right)\left(u U_{1} V^{\prime}-v V_{1} U^{\prime}\right)=0 .
\end{aligned}
$$

Writing $u^{2}=v^{2}=\alpha$, we have from (15) $U(\alpha)+V(\alpha)=0$, and hence may write $U(\alpha)=-V(\alpha)=f(\alpha)$.

Replacing $u^{2}$ by $\alpha+h, v^{2}$ by $\alpha$ and allowing for the change of variables where the derivatives $U^{\prime}$ and $V^{\prime}$ appear, we have $f^{\prime 2}\left(k^{4}-l^{4}\right)=0$, the equation obtained by equating to zero the terms in (15) which are independent of $h$. 
The case where the function vanishes is of no interest, and by equating to zero the coefficients of other powers of $h$ we can eliminate the case $k^{2}+l^{2}=0$, leaving as the only possible solution

$$
k^{2}=l^{2} .
$$

In the preceding work we assumed that the functions $U_{1}$ and $V_{1}$ were even. If this is not so, we may divide equation (14) into three parts, one composed of all terms containing $U_{1}$ and $V_{1}$, another of all remaining terms which change their sign with either variable, and the third of all the other terms.

Evidently the second part is equal to the difference between the two expressions obtained by replacing $u$ by $-u$ in the first. Taking the equation thus formed, and treating it exactly as in the preceding case, we arrive at the same conclusion with regard to the constants.

Excluding cases $1^{\circ}$ to $6^{\circ}$, then, the form (I) reduces to

$$
\text { (I) } \quad \overline{d s}^{2}=\left(\frac{v-u}{v+u}\right)^{2 k}\left(\frac{d u^{2}}{U^{2}}+\frac{d v^{2}}{V}\right) \quad \text { or } \quad(\mathrm{I})^{\prime \prime} \quad \overline{d s^{2}}=\left(v^{2}-u^{2}\right)^{2 k}\left(\frac{d u^{2}}{U}+\frac{d v^{2}}{V}\right) \text {. }
$$

To every surface whose linear element has the form (I)' corresponds another whose linear element has the form $(\mathrm{I})^{\prime \prime}$, and vice versa.*

We have discussed all surfaces corresponding to $(\mathrm{I})^{\prime \prime}$ in a previous paper $\dagger$ and from that discussion and the relation $(\mathrm{I})^{\prime}$ and $(\mathrm{I})^{\prime \prime}$ conclude that all solutions of $(\mathrm{I})^{\prime}$ have already been considered in $\S 3$.

Of the six exceptional cases which we have excluded up to this point, only $5^{\circ}$ (or $6^{\circ}$ ) in the light of the preceding work of this paper needs consideration, and here only the case in which neither of the constants vanishes.

Let us consider for example the case $l=1, k \neq 0$ or \pm 1 . The coefficient of $(U+V)^{2}$ in $(14)$ is now divisible by $(u+v)^{2}$ but not by $(u-v)^{2}$, and hence we still have

$$
U(\alpha)=-V(\alpha)=f(\alpha) .
$$

Replacing $u$ by $(\alpha+h)$ and $v$ by $\alpha$, and expanding in powers of $h$, we have, on equating the coefficient of $h$ to zero,

$$
4 k(k-1)(2 k+1) f^{\prime 2}=0 ;
$$

hence the only possible solutions are those already considered.

The form (I), given at the beginning of this article, is therefore characteristic of the surfuces discussed in \$§4-7.

Purdue University.

* Proof similar to that used in 86.

† Loo. cit., p. 416. 